\title{
Oxidative Rearrangement Processes in the Biosynthesis of Gilvocarcin V
}

Tao Liu, Carsten Fischer, Claus Beninga, and Jürgen Rohr*

Supporting information 


\section{Methods}

\section{Bacterial strains, cosmids and culture conditions}

Cosmid $\operatorname{cosG9B3}{ }^{1}$ is pOJ446-derived and contains the entire gilvocarcin gene cluster.

Streptomyces lividans TK24 was routinely cultured on M2 agar plates containing $1.5 \%$ agar, $0.4 \%$ glucose, $1 \%$ Malt extract, $0.4 \%$ yeast extract and $0.1 \% \mathrm{CaCO}_{3}$ up to sporulation, and spores were stocked in $20 \%$ glycerol and used for conjugation. $2 \times$ YT broth, containing $1.6 \%$ tryptone, $1 \%$ yeast extract and $0.5 \%$ $\mathrm{NaCl}(\mathrm{pH} 7.0)$, was used during conjugation.

Escherichia coli XL1 Blue MRF' (Stratagene) was used for propagation of plasmids, cosmids and grown in liquid at $220 \mathrm{rpm}$ or on solid Luria-Bertani medium (containing $1 \%$ tryptone, $0.5 \%$ yeast extract, $1 \% \mathrm{NaCl} ; \mathrm{pH} 7.0 ; 1.5 \%$ agar) at $37^{\circ} \mathrm{C}$.

The REDIRECT ${ }^{\circledR}$ technology kit containing E. coli ET12567, E. coli ET12567/pUZ8002, E.coli BW25113, pKD20, plJ790 and pCP20 was a gift from Plant Bioscience Ltd. (Nowrich, United Kingdom).

Apramycin $(50 \mu \mathrm{g} / \mathrm{mL})$, chloramphenicol $(25 \mu \mathrm{g} / \mathrm{mL})$, nalidixic acid $(25 \mu \mathrm{g} \mathrm{mL} / \mathrm{L})$, carbenicillin $(100 \mu \mathrm{g} / \mathrm{mL})$ and kanamycin $(50 \mu \mathrm{g} / \mathrm{mL})$ were used for selection of recombinant strains.

\section{DNA isolation, manipulation and cloning}

Standard procedures for DNA isolation and manipulation were performed according to Sambrook and Russel ${ }^{2}$ and Kieser et al. ${ }^{3}$ Isolation of DNA fragments from agarose gels and purification of PCR products were carried out using the QIA quick ${ }^{\circledR}$ Gel Extraction kit (Qiagen, California, USA) following the manufacturer's instructions. Isolation of the mutated derivatives of cosmidG9B3 was carried out using ion exchange columns (Nucleobond AX kits, MachereyNagel, PA, USA) according to the manufacturer's protocol.

\section{Inactivation of gilOI and gilOIV}


The inactivation of gilOI or gilOIV in cosG9B3 (containing the apramycin (Apr)resistance cassette and the entire gilvocarcin $V$ gene cluster) was modified using the PCR-targeting method described by Gust et al. ${ }^{4}$ The coding sequence of gilOI and gilOIV, respectively, was replaced by the chloramphenicol $(\mathrm{CHL})$ resistance cassette through double crossover. In the following FLP-mediated excision of the disruption cassette, mutants were selected for Apr-resistance and CHL-sensitivity (Figure 1).

A disruption cassette containing the chloramphenicol resistance gene flanked by FRT sites was generated from plJ790 by PCR using primer pair CHL_for and CHL_rev including FRT sites as plJ773/plJ778/plJ780, which were underlined (see table 1), and the cassette was cloned into the TOPO pCRß-Blunt Vector following manufactures' instruction (Zero Blunt ${ }^{\circledR}$ TOPO PCR Cloning Kit, Invitrogen, CA, USA) allowing an EcoR V/Hindlll fragment for use as the template for further PCR reaction of inactivation. The disruption gene cassette was amplified using the above gene cassette combined with primer pair GilOI_for and GilOI_rev (see table 1). Underlined letters represent $39 \mathrm{nt}$ homologous extensions to the DNA regions immediately upstream and downstream of gilOI, respectively, including the putative start and stop codons of gilOI. Underlined letters for gilOIV represent $39 \mathrm{nt}$ homologous extensions to the DNA regions immediately upstream and sequence from 1269 to 1307 (stop codon -45nt) in order to prevent truncating downstream genes because gilOIV overlapped with downstream gene. The cassette was introduced into E. coli BW25113/pKD20, containing cosmid G9B3 (apramycin-resistant), which included the entire biosynthetic gene cluster of gilvocarcin V. The disrupted cosmid G9B3 with chloramphenicol was introduced into $\mathrm{XL}-\mathrm{Blue} \mathrm{MRF}$ /pcp20 to remove the central part of disruption cassette. Apramycin-resistant, chloramphenicol-sensitive colonies were identified by replica plating and verified by PCR analysis using pair of primers GilOI_Ctrl_forw and GilOI_Ctrl_rev (for gilOIV, GilOIV_Ctrl_forw and GilOIV_Ctrl_rev, see table 1). While the original gilOI and gilOIV PCR products from cosG9B3 showed products of $1627 \mathrm{bp}$ and $1509 \mathrm{bp}$, respectively, the mutants with the chloramphenicol resistance gene gave PCR-products of 1154 
bp and 1250bp, respectively. After FLP-mediated excision of the disruption cassette, PCR products of $208 \mathrm{bp}$ and $304 \mathrm{bp}$, respectively, were obtained (Figure 2). The mutated cosG9B3 was introduced into Streptomyces lividans TK24 by conjugation from E. coli ET12567 carrying the non-transmissible pUZ8002 as described by Kieser et al. ${ }^{3}$

\section{Table 1}

Oligonucleotide primers used in PCR generating disruption cassette containing chloramphenicol resistance gene and analyzing inactivated mutants

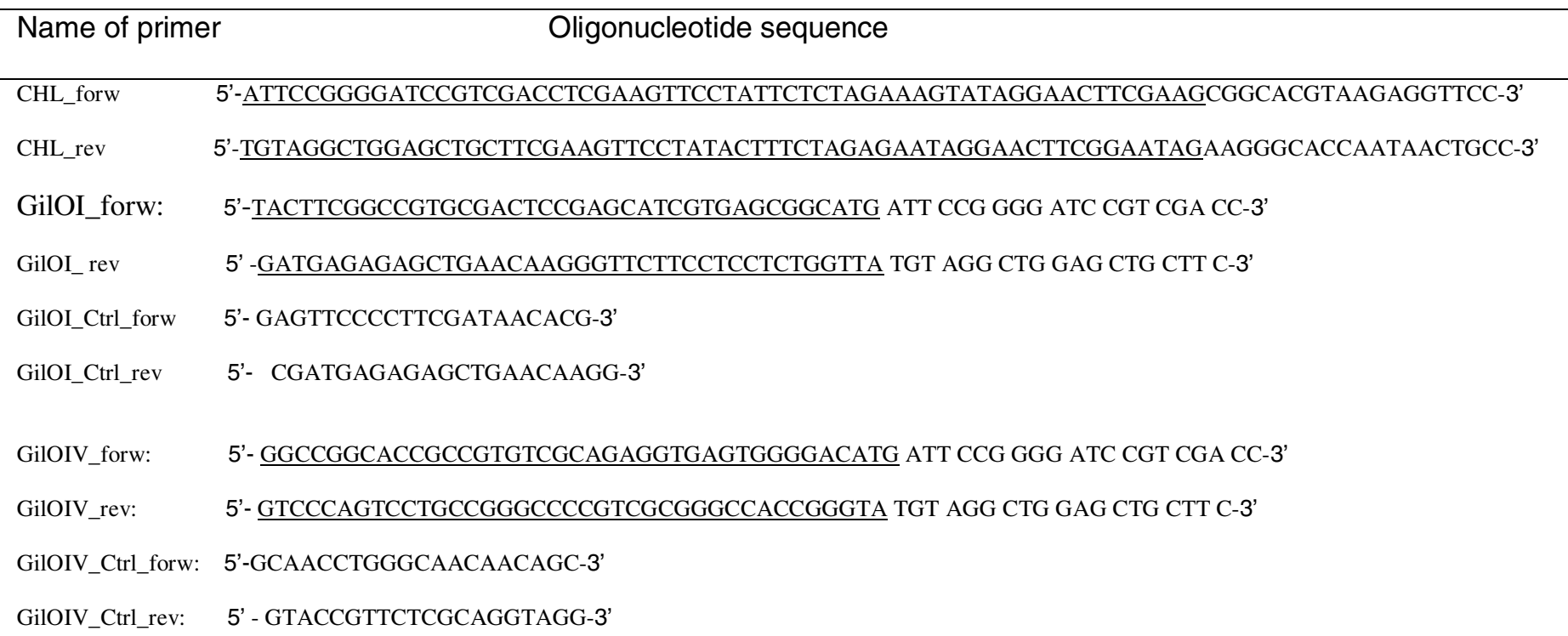


Figure 1

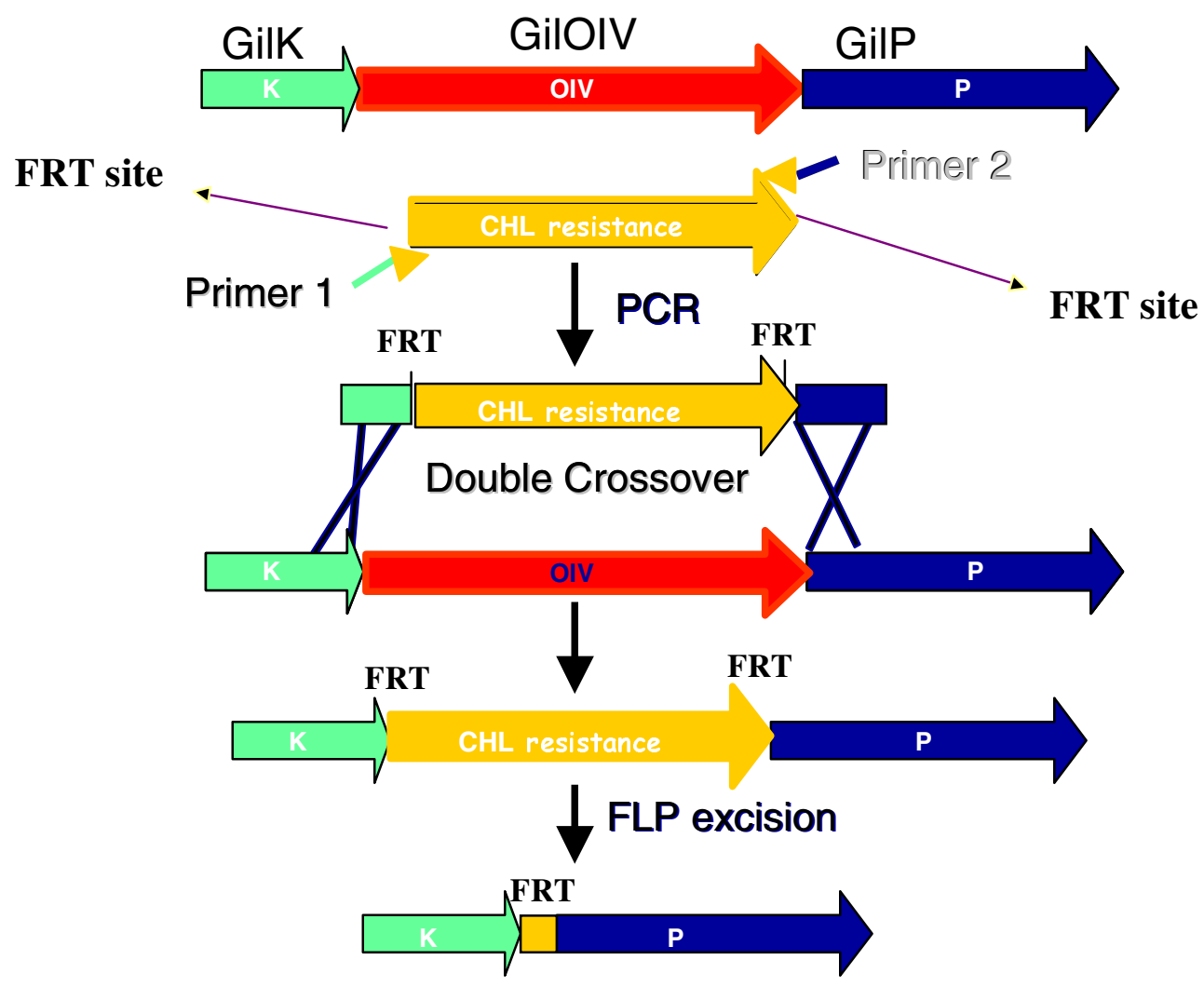

Figure 1: Application the Redirect PCR Targeting System in CosG9B3 for the inactivation of gilOIV. FRT: FLP recognition target, CHL: chloramphenicol resistance gene; primer 1: gilOIV_rev; primer2: gilOIV_forw. The inactivation of gilOI was accomplished analogously. 
Figure 2: Gene Deletion of gilOI and gilOIV

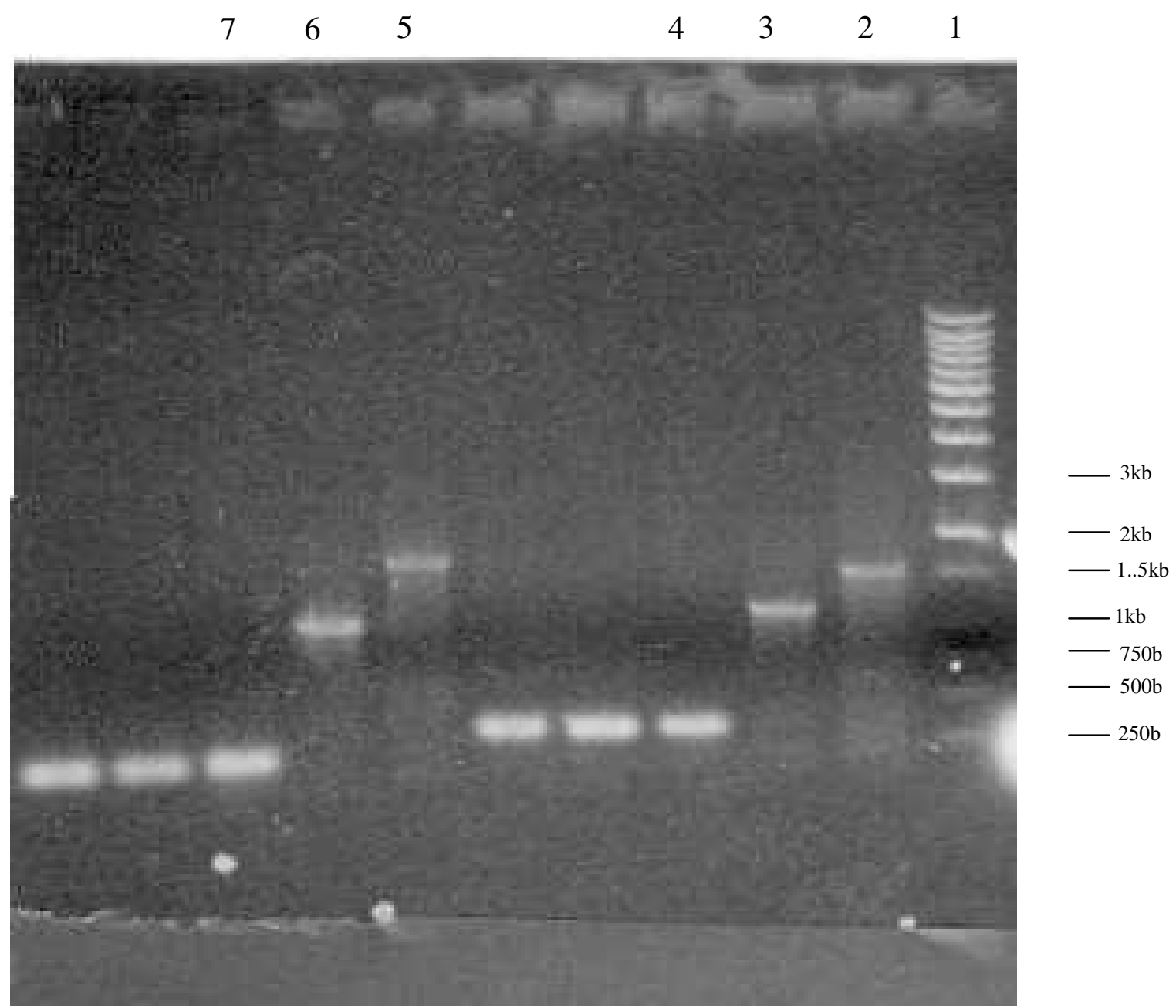

Figure 2: PCR control reactions showing the replacement of gilOl and gilOIV with the chloramphenicol resistance cassette and subsequent scission with FLP recombinase $(0.7 \%$ agarose gel)

1: $1 \mathrm{~kb}$ DNA ladder; 2: original gilOIV; 3: gilOIV in cosG9B3 is replaced by the chloamphenicol resistance gene cassette; 4: chloramphenicol resistance gene is excised from the disrupted gilOIV mutant; 5: original gilOl; 6: gilOI in cosG9B3 is replaced by the chloramphenicol resistance gene cassette; 7: the chloramphenicol resistance gene is excised from the disrupted gilOl-mutant. 
Analysis, isolation and characterization of secondary metabolites in $S$.
lividans TK24 (cosG9B3) strains

All S. lividans TK24 (cosG9B3) mutants were cultured in SG medium (20 g/L glucose, $10 \mathrm{~g} / \mathrm{L}$ soy peptone, $2 \mathrm{~g} / \mathrm{L} \mathrm{CaCO}_{3}, 1 \mathrm{mg} / \mathrm{L} \mathrm{CoCl} 2 ; \mathrm{pH} 7.2$ prior to sterilization) supplemented with apramycin. This preculture was grown for 1 day at $30{ }^{\circ} \mathrm{C}$ and $220 \mathrm{rpm}$, subsequently used to inoculate the main culture of the same composition, which was harvested after 4 days of shaking as above. The culture broth was extracted three times with equal volumes of ethyl acetate. Extracts were dried in vacuo, dissolved in methanol and examined by HPLC-MS.

Purification was achieved by semi-preparative HPLC. HPLC/MS was performed on a Waters Alliance 2695 system with Waters 2996 photodiode array detector and a Micromass ZQ 2000 mass spectrometer equipped with an $\mathrm{APCl}$ ionization probe (solvent $\mathrm{A}=0.1 \%$ formic acid in $\mathrm{H}_{2} \mathrm{O}$; solvent=acetonitrile; flow rate $=0.5 \mathrm{~mL} / \mathrm{min} ; 0-6 \mathrm{~min} 75 \% \mathrm{~A}$ and $25 \% \mathrm{~B}$ to $100 \% \mathrm{~B}$ [linear gradient], $7.5-10$ min $75 \%$ A and $25 \%$ B). Semi-preparative HPLC was run on a Waters Delta 600 instrument with a Waters 996 photodiode array detector (Solvent $A=0.1 \%$ trifluoroacetic acid in $\mathrm{H}_{2} \mathrm{O}$; solvent $\mathrm{B}=$ acetonitrile; $0-20 \mathrm{~min} 75 \% \mathrm{~A}$ and $25 \% \mathrm{~B}$ to $100 \%$ B [linear gradient], $20-23 \mathrm{~min} 100 \% \mathrm{~B}, 23-24 \mathrm{~min} 100 \% \mathrm{~B}$ to $75 \% \mathrm{~A}$ and $25 \%$ B [linear gradient], 24-32.5min $75 \% \mathrm{~A}$ and $25 \% \mathrm{~B}$. The columns used for HPLC were Waters Symmetry $\mathrm{C}_{18}, 4.6 \times 50 \mathrm{~mm}$, particle size $5 \mu \mathrm{m}(\mathrm{HPLC} / \mathrm{MS})$, and Waters Symmetry Prep ${ }^{\mathrm{TM}} \mathrm{C}_{18}, 19 \times 150 \mathrm{~mm}$, particle size $7 \mu \mathrm{m}$ (semi-prep. HPLC).

\section{Homorabelomycin (5)}

Yield: $2.5 \mathrm{mg} / \mathrm{L} ; \mathrm{R}_{\text {rel }}=5.37 \mathrm{~min}$ $\mathrm{MW}=352 \mathrm{~g} / \mathrm{mol}\left(\mathrm{C}_{20} \mathrm{H}_{16} \mathrm{O}_{6}\right)$

Positive mode ESI-MS: $\mathrm{m} / \mathrm{z}=353$ (high resolution calc for $\mathrm{C}_{20} \mathrm{H}_{17} \mathrm{O}_{6}$ : 353.1020 , found 353.1014)

UV maxima (from HPLC-diode array): 230 (69\%), 267 (100\%), 428 (29\%)<smiles>CCC1(O)CC(=O)c2c(cc(O)c3c2C(=O)c2cccc(O)c2C3=O)C1</smiles> 
${ }^{1} \mathrm{H}-\mathrm{NMR}(300 \mathrm{MHz}$, Acetone-D6, TMS)
${ }^{13}$ C-NMR (100.6 MHz, Acetone-D6, TMS

\begin{tabular}{|c|c|c|c|}
\hline Position & $\delta^{1} \mathrm{H}$ [ppm] & Multiplicity & $\delta{ }^{13} \mathrm{C}$ [ppm] \\
\hline 1 & & & 196.1 \\
\hline $\begin{array}{l}2_{e q} \\
2_{a x}\end{array}$ & $\begin{array}{l}2.76 \\
2.96\end{array}$ & $\begin{array}{c}\text { dd }(15,2) \\
d(15)\end{array}$ & 52.2 \\
\hline 3 & & & 74.5 \\
\hline $3-\mathrm{OH}$ & 3.95 & s & \\
\hline $\begin{array}{l}4_{\text {eq }} \\
4_{\text {ax }}\end{array}$ & $\begin{array}{l}3.09 \\
3.21\end{array}$ & $\begin{array}{c}\text { dd }(17,2) \\
d(17)\end{array}$ & 42.8 \\
\hline $4 a$ & & & 153.2 \\
\hline 5 & 7.13 & $\mathrm{~s}$ & 122.6 \\
\hline 6 & & & 162.7 \\
\hline $6-\mathrm{OH}$ & 12.2 & $s$ & \\
\hline $6 a$ & & & 117.5 \\
\hline 7 & & & 193.7 \\
\hline $7 a$ & & & 116.3 \\
\hline 8 & & & 164.2 \\
\hline $8-\mathrm{OH}$ & 11.7 & $\mathrm{~s}$ & \\
\hline 9 & 7.33 & $\mathrm{dd}(8,1)$ & 124.3 \\
\hline 10 & 7.83 & dd $(8,8)$ & 138.7 \\
\hline 11 & 7.57 & $\mathrm{dd}(8,1)$ & 119.9 \\
\hline $11 a$ & & & 137.0 \\
\hline 12 & & & 183.9 \\
\hline $12 a$ & & & 138.8 \\
\hline $12 b$ & & & 130.8 \\
\hline 13 & 1.73 & $\mathrm{q}(7.5)$ & 35.9 \\
\hline 14 & 1.03 & $\mathrm{t}(7.5)$ & 7.9 \\
\hline
\end{tabular}

\section{Rabelomycin (6)}

Yield: $<0.5 \mathrm{mg} / \mathrm{L} ; \mathrm{R}_{\mathrm{rel}}=4.91 \mathrm{~min}$

$\mathrm{MW}=338 \mathrm{~g} / \mathrm{mol}\left(\mathrm{C}_{19} \mathrm{H}_{14} \mathrm{O}_{6}\right)$

UV maxima (from HPLC-diode array): 230 (69\%), 267 (100\%), 428 (29\%)

The isolated compound was identical with respect of TLC, HPLC-MS and ${ }^{1} \mathrm{H}-$ NMR with previously isolated and literature-known compound rabelomycin., 


\section{Pregilvocarcin V-o-quinone (7)}

Yield: $10 \mathrm{mg} / \mathrm{L} ; \mathrm{R}_{\text {rel }}=7.13 \mathrm{~min}$

$\mathrm{MW}=334 \mathrm{~g} / \mathrm{mol}\left(\mathrm{C}_{20} \mathrm{H}_{14} \mathrm{O}_{5}\right)$

Positive mode APCI-MS: $\mathrm{m} / \mathrm{z}=335$; HR-EI-MS: $\mathrm{m} / \mathrm{z}=334$ (calcd for $\mathrm{C}_{20} \mathrm{H}_{14} \mathrm{O}_{5}$

334.0841, found 334.833)

UV maxima (from HPLC-diode array): 237 (90\%), 248 (88\%), 261 (100\%), 450 $(18 \%)$

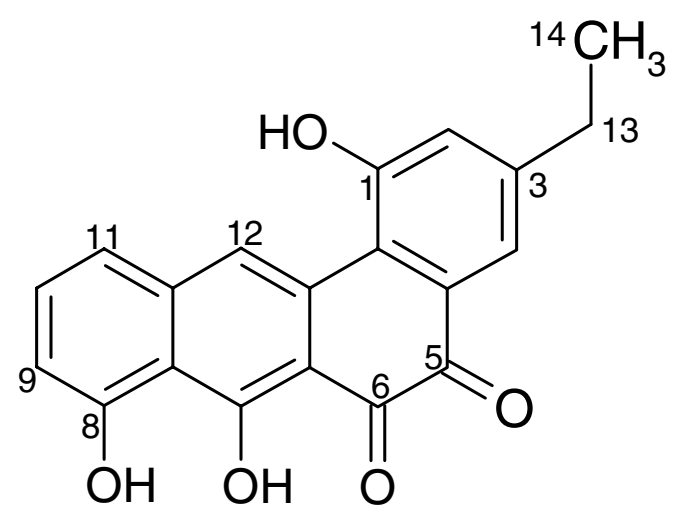

${ }^{1} \mathrm{H}-\mathrm{NMR}$ (400 MHz, DMSO-D6, TMS)

${ }^{13}$ C-NMR (100 MHz, DMSO-D6, TMS)

\begin{tabular}{|c|c|c|c|c|}
\hline $\begin{array}{c}\text { Position } \\
\text { number }\end{array}$ & ${ }^{1} \mathrm{H} \delta$ & Multiplicity & ${ }^{13} \mathrm{C} \delta$ & $\begin{array}{c}\text { HMBC } \\
\text { correlation }\end{array}$ \\
\hline 1 & $-\mathrm{OH} 11.00$ & $\mathrm{~S}$ & 157.5 & \\
\hline 2 & 7.17 & $d(1.6)$ & 122.9 & $4,12 b, 13$ \\
\hline 3 & & & 145.8 & \\
\hline 4 & 7.47 & $\mathrm{~d}(1.6)$ & 119.0 & $2,5,12 b, 13$ \\
\hline $4 a$ & & & 116.6 & \\
\hline 5 & & & 182.0 & \\
\hline 6 & & & 186.4 & \\
\hline $6 a$ & & & 113.4 & \\
\hline 7 & $-\mathrm{OH} 16.40$ & br s & 168.6 & \\
\hline $7 \mathrm{a}$ & & & 112.2 & \\
\hline 8 & $-\mathrm{OH} 11.30$ & br s & 161.3 & \\
\hline 9 & 6.93 & $\mathrm{~d}(8.0)$ & 114.2 & $7 a, 11$ \\
\hline 10 & 7.63 & $\mathrm{t}(8.0)$ & 137.6 & $8,11 \mathrm{a}$ \\
\hline 11 & 7.20 & $\mathrm{~d}(8.0)$ & 122.3 & $7 a, 9$ \\
\hline $11 \mathrm{a}$ & & & 140.2 & \\
\hline 12 & 8.82 & $\mathrm{~S}$ & 126.3 & $6 a, 11,12 b$ \\
\hline $12 \mathrm{a}$ & & & 132.5 & \\
\hline $12 b$ & & & 118.4 & \\
\hline 13 & 2.63 & $q(7.6)$ & 28.1 & $2,3,4,14$ \\
\hline 14 & 1.20 & $\mathrm{t}(7.6)$ & 15.3 & 3,13 \\
\hline
\end{tabular}




\section{Pregilvocarcin M-o-quinone (8)}

Yield: $1 \mathrm{mg} / \mathrm{L} ; \mathrm{R}_{\text {rel }}=6.76 \mathrm{~min}$

$\mathrm{MW}=320 \mathrm{~g} / \mathrm{mol}\left(\mathrm{C}_{19} \mathrm{H}_{12} \mathrm{O}_{5}\right)$

Positive mode APCI-MS: $\mathrm{m} / \mathrm{z}=321$

UV maxima (from HPLC-diode array): 237 (90\%), 248 (88\%), 261 (100\%), 450 $(18 \%)$

\section{2,3-dehydro-homo-UWM6 (9)}

Yield: $1 \mathrm{mg} / \mathrm{L} ; \mathrm{R}_{\text {rel }}=5.53$

$\mathrm{MW}=338 \mathrm{~g} / \mathrm{mol}\left(\mathrm{C}_{20} \mathrm{H}_{18} \mathrm{O}_{5}\right)$

Positive mode ESI-MS: $\mathrm{m} / \mathrm{z}=361$ (high resolution: calc. for $\mathrm{C}_{20} \mathrm{H}_{18} \mathrm{O}_{5} \mathrm{Na}$ : 361.1046, found 361.1044 )

UV maxima (from HPLC-diode array): 225 (85\%), 265 (100\%), 406 (24\%)<smiles>CCC1=CC(=O)C2c3cc4cccc(O)c4c(O)c3C(=O)CC2(O)C1</smiles>

${ }^{1} \mathrm{H}-\mathrm{NMR}$ (400 MHz, DMSO-D6, TMS)

${ }^{13}$ C-NMR (100 MHz, DMSO-D6, TMS)

\begin{tabular}{|l|c|c|c|l|}
\hline $\begin{array}{l}\text { Position } \\
\text { number }\end{array}$ & ${ }^{1} \mathrm{H} \delta$ & $\begin{array}{c}\text { Multiplicity } \\
(\mathrm{J} / \mathrm{Hz})\end{array}$ & ${ }^{13} \mathrm{C} \delta$ & $\begin{array}{l}\text { HMBC } \\
\text { correlation }\end{array}$ \\
\hline 1 & & & 199.1 & \\
\hline 2 & 5.81 & $\mathrm{~s}$ & 123.0 & 13 \\
\hline 3 & & & 164.8 & \\
\hline 4 & 2.32 and 2.42 & br d (17) and d (17) & $40^{\mathrm{a}}$ & \\
\hline $4 \mathrm{a}$ & $-\mathrm{OH} 5.14$ & & 72.9 & \\
\hline 5 & $\sim 3.35^{\mathrm{b}}$ & & 63.7 & \\
\hline 6 & & & 192.9 & \\
\hline $6 \mathrm{a}$ & & & 111.5 & \\
\hline 7 & $-\mathrm{OH} 15.9$ & & 177.0 & \\
\hline $7 \mathrm{a}$ & & & 118.5 & \\
\hline 8 & & & 163.3 & \\
\hline 9 & 6.25 & $\mathrm{~d}(8)$ & 108.0 & $7 \mathrm{a}, 11$ \\
\hline 10 & 7.12 & $\mathrm{t}(8)$ & 131.1 & $8,11 \mathrm{a}$ \\
\hline 11 & 6.61 & $\mathrm{~d}(8)$ & 114.5 & $9,7 \mathrm{a}$ \\
\hline
\end{tabular}




\begin{tabular}{|l|c|c|c|l|}
\hline $11 \mathrm{a}$ & & & 138.8 & \\
\hline 12 & 6.05 & & 108 & $6 \mathrm{a}, 7 \mathrm{a}$ \\
\hline $12 \mathrm{a}$ & & & 135.9 & \\
\hline $12 \mathrm{~b}$ & 3.50 & $\mathrm{~s}$ & 59.7 & $1,4 \mathrm{a}, 12 \mathrm{a}$ \\
\hline 13 & 2.11 & br q (7.6) & 30.2 & $2,3,14$ \\
\hline 14 & 0.97 & $\mathrm{t}(7.6)$ & 11.5 & 3,13 \\
\hline
\end{tabular}

${ }^{a}$ obscured by solvent DMSO; ${ }^{b}$ obscured by water

\section{2,3-dehydro-UWM6 (10)}

$\mathrm{R}_{\mathrm{rel}}=5.01$

Yield: $0.2 \mathrm{mg} / \mathrm{L}$

$\mathrm{MW}=324 \mathrm{~g} / \mathrm{mol}\left(\mathrm{C}_{29} \mathrm{H}_{16} \mathrm{O}_{5}\right)$

Positive mode APCI-MS: $\mathrm{m} / \mathrm{z}=325$ (low resolution)

UV maxima (from HPLC-diode array): 225 (85\%), 265 (100\%), 406 (24\%)

\section{Incorporation experiments with ${ }^{18} \mathrm{O}$-labeled precursors}

The fermentation of Streptomyces griseoflavus Gö 3592 was carried out in a $1 \mathrm{~L}-$ fermentor using SG medium (20 g/L glucose, $10 \mathrm{~g} / \mathrm{L}$ soy peptone, $2 \mathrm{~g} / \mathrm{L} \mathrm{CaCO}$, $1 \mathrm{mg} / \mathrm{L} \mathrm{CoCl}$ ) at $30{ }^{\circ} \mathrm{C}$ and $300 \mathrm{rpm}$ for $108 \mathrm{~h}$. For the fermentation under ${ }^{18} \mathrm{O}$ enriched atmosphere, the $1 \mathrm{~L}$-fermentor was connected to a closed system apparatus described previously. ${ }^{7}$ Gilvocarcin V (GV) was isolated by extraction of the mycelium with $500 \mathrm{~mL}$ acetone. Removal of the acetone in vacuo, followed by extraction of the water residue with $300 \mathrm{~mL}$ chloroform and evaporation to dryness, yielded a solid residue. Most impurities were removed by treatment of this residue with methanol, since GV is not soluble in methanol, and could be filtered off as a $90 \%$-enriched residue. Final purification was achieved by HPLC (see above) to yield 10 to $30 \mathrm{mg}$ pure gilvocarcin V. For the incorporation with ${ }^{18} \mathrm{O}$-labeled acetate, $1 \mathrm{~g}$ of $\left[1-{ }^{13} \mathrm{C},{ }^{8} \mathrm{O}_{2}\right]$-acetate was fed in three portions at $36 \mathrm{~h}$, $48 \mathrm{~h}$ and $60 \mathrm{~h}$ of the fermentation. ${ }^{18} \mathrm{O}$-labeled oxygen atoms were detected by an upfield shift the ${ }^{13} \mathrm{C}$-NMR spectrum, ${ }^{8,9}$ which was for $\mathrm{C}-1: \Delta \delta=0.01 \mathrm{ppm}$; for C$10: \Delta \delta=0.03 \mathrm{ppm}$; and for C-12: $\Delta \delta=0.02 \mathrm{ppm}$. For the fermentation under ${ }^{18} \mathrm{O}-$ enriched atmosphere, the normal atmosphere was replaced at the beginning of the gilvocarcin production (after $36 \mathrm{~h}$ ) by an atmosphere consisting of $80 \%$ nitrogen, and $20 \%{ }^{18} \mathrm{O}_{2}$ (50\% enriched).

\section{References}

(1) Fischer, C.; Lipata, F.; Rohr, J. The Complete Gene Cluster of the Antitumor Agent Gilvocarcin V and Its Implication for the Biosynthesis of the Gilvocarcins J. Am. Chem. Soc. 2003, 125, 7818-7819.

(2) Sambrook, J.; Russel, D. W. Molecular Cloning. A Laboratory Manual, 3rd ed.; 3rd ed.; Cold Spring Harbor Laboratory Press: Cold Spring Harbor, NY, 2001. 
(3) Kieser, T.; Bibb, M. J.; Buttner, M. J.; Chater, K. F.; Hopwood, D. A. Practical Streptomyces Genetics; The John Innes Foundation: Norwich, UK, 2000.

(4) Gust, B.; Challis, G. L.; Fowler, K.; Kieser, T.; Chater, K. F. PCR-trageted Streptomyces gene replacement identifies a protein domain needed for biosynthesis of the sesquiterpene soil odor geosmin Proc. Natl. Acad. Sci. USA 2003, 100, 1541-1546.

(5) Rix, U.; Remsing, L. L.; Hoffmeister, D.; Bechthold, A.; Rohr, J. Urdamycin L: A novel metabolic shunt product that provides evidence for the role of the $u r d \mathrm{M}$ gene in the urdamycin A biosynthetic pathway of Streptomyces fradiae TÜ2717 Chembiochem 2003, 4, 109-111.

(6) Krohn, K.; Khanbabaee, K. First total synthesis of (+/-)-rabelomycin Angewandte Chemie International Edition in English 1994, 33, 99-100.

(7) Udvarnoki, G.; Henkel, T.; Machinek, R.; Rohr, J. Biosynthetic Origin of the Oxygen Atoms of Aquayamycin: Aspects for the Biosynthesis of the Urdamycin Family for Aquayamycin-Containing Angucycline Antibiotics in General J. Org. Chem. 1992, 57, 1274-1276.

(8) Vederas, J. C. Biosynthetic Studies Using 180 Isotope Shifts in 13C Nuclear Magnetic Resonance Can. J. Chem. 1982, 60, 1637-1642.

(9) Van Etten, R. L. In Proceedings of the Second International Symposium on Synthesis and Applications of Isotopically Labeled Compounds 1985, Kansas City, MO. U.S.A., 3-6 Sept. 1985; Muccino, R. R., Ed.; Elsevier Science Publishers: Amsterdam, 1986, pp 257-262. 